\title{
Aplicação dos conceitos de Controle Estatístico de Processo (CEP) em uma indústria de fundição do Norte Catarinense
}

\section{Application of the concepts of Statistical Control of Process (CEP) in an industry of casting of the Catarinense North}

\section{Gueibi Peres Souza Msc.}

Doutorando do PPGEP

Universidade Federal de Santa Catarina

Departamento de Engenharia de Produção e Sistemas

Rod. Admar Gonzaga, 1623, Apto. 102 B1 2 - Itacorubi, Fpolis - SC - Brasil 88034001

(48) 331-7027 gueibi@deps.ufsc.br

\section{Manoel Domingos Filho Msc.}

Doutorando do PPGEP

Universidade Federal de Santa Catarina

Departamento de Engenharia de Produção e Sistemas

Rua José Henrique Veras 431 - Lagoa da Conceição, Fpolis - SC - Brasil 88032-060

(48) 331-7027 manoeld@deps.ufsc.br

\author{
Robert W. Samohyl, Ph.D \\ Professor do PPGEP \\ Universidade Federal de Santa Catarina \\ Departamento de Engenharia de Produção e Sistemas
}

Rod. Tertuliano de Brito Xavier, 23252 Colinas de S. Francisco, Apto. 101 Bl Yrupê

- Canasvieiras, Fpolis - SC - Brasil 88054-601

(48) 331-7027 samohyl@deps.ufsc.br 


\title{
RESUMO
}

O objetivo desse artigo é realizar um estudo de caso em uma indústria de fundição, localizada no Estado de Santa Catarina, aplicando conceitos básicos de Controle Estatístico de Processo (CEP). Mais precisamente, nosso objetivo consiste em analisar a qualidade e a capacidade de produção da indústria, visando identificar particularidades e características do processo, assim como oportunidades de melhorias úteis aos operadores e responsáveis pelo monitoramento do processo na empresa, ou mesmo para outras pessoas que trabalhem e lidem com processos similares. Nesse estudo, utilizamos alguns dos principais tipos de gráficos de controle já consagrados na literatura, tais como o gráfico de controle para observações individuais; o gráfico da amplitude móvel; o gráfico da média móvel exponencialmente ponderada e o gráfico da soma cumulativa. $\mathrm{O}$ trabalho limita-se apenas a realizar análises estatísticas sobre os dados fornecidos para estudo e possivelmente construir conjecturas baseadas nas informações transmitidas pela empresa, esperando e acreditando na fidedignidade e veracidade das mesmas, sem, no entanto desprezar toda e qualquer informação e/ou inferência adquirida a partir de um olhar mais técnico científico e não tanto empírico dos dados. Nesse artigo, nós não temos a pretensão de desenvolver métodos e modelos estatísticos mais robustos para realizar tal estudo.

Palavras-chave: Gráficos da Soma Cumulativa; Gráfico da Média Móvel Ponderada Exponencialmente; Índice de Capacidade de Processo.

\begin{abstract}
This paper aims to apply the zip code concept in real data of a casting industry in Santa Catarina, in order to identify features and particularities of this process, as well as improving useful qualities for employees who monitories the company procedure, or even other people who work and deal with similar processes. The paper uses basically the construction of control charts, which are more suitable to the sample collecting features made in the company, besides calculating its capacity, what brings interesting issues for the main identified problems which must be improved. The paper limits to perform statistical analysis and consequently to build conjectures based on the information provided by the company, believing in the veracity of it, without setting aside any information or inference acquired through a more scientific, technical approach, not just empirical, of the data. This is the way theoretical concepts found in literature and practical procedures watched in the company will be discussed, always aiming for validations that indicate the route of new researches, as well as exploring new opportunities decurrently of the adoption of other procedures.
\end{abstract}

Key Words: Cumulative-Sum Control Chart; Exponentially Weighted Moving Average Control Chart; Process Capability Ratios. 


\section{INTRODUÇÃO}

Uma indústria de fundição que produz blocos para veículos automotores, situada no Norte do Estado de Santa Catarina, fornece seus produtos a grandes nomes da indústria automotiva mundial, portanto, atua em um mercado bastante competitivo, onde a qualidade é um aspecto qualificador e também ganhador de pedidos. Aplicando-se conceitos de controle estatístico de processo (CEP), por exemplo, em produtos tidos como estratégicos pela empresa, é possível se identificar diretrizes que levem a uma maior qualidade a ser percebida por parte do cliente.

Um dos produtos considerados como estratégico, por já estar consolidado no mercado, sendo inclusive tradicional na fundição da empresa, é um determinado bloco de motor para caminhões a diesel. Um dos aspectos em que é medida sua qualidade é através de sua dureza, ou seja, de seu grau de resistência à tração, tendo como unidade de medida o Mega Pascal (Mpa).

O processo de avaliação da qualidade das unidades produzidas requer um teste destrutivo do corpo de prova. O mesmo exige que o bloco seja cortado, para que se retire o(s) corpo(s) de prova que serão usinados e em seguida submetidos a um ensaio de tração com limite mínimo de especificação de 230 Mpa.

Devido a esta particularidade do processo de inspeção, a coleta de amostras se dá de forma unitária. Outros aspectos que corroboram para que se adote este tipo de amostragem é o fato das grandes dimensões do bloco, o que dificulta seu manuseio, além do fato de que ele só pode ser submetido ao ensaio de tração após estar resfriado (variando entre 45 e 240 minutos após sua fundição, dependendo das condições locais de temperatura), o que inviabiliza uma inspeção antes que todo o lote seja produzido.

O processo de inspeção se dá sempre com o primeiro bloco do lote que é produzido, se sua propriedade de dureza respeitar o valor crítico o lote é aceito, caso contrário segue-se um procedimento que admite a existência de problemas com o(s) corpo(s) de ensaio, o que pode ser visualizado na figura 1. 


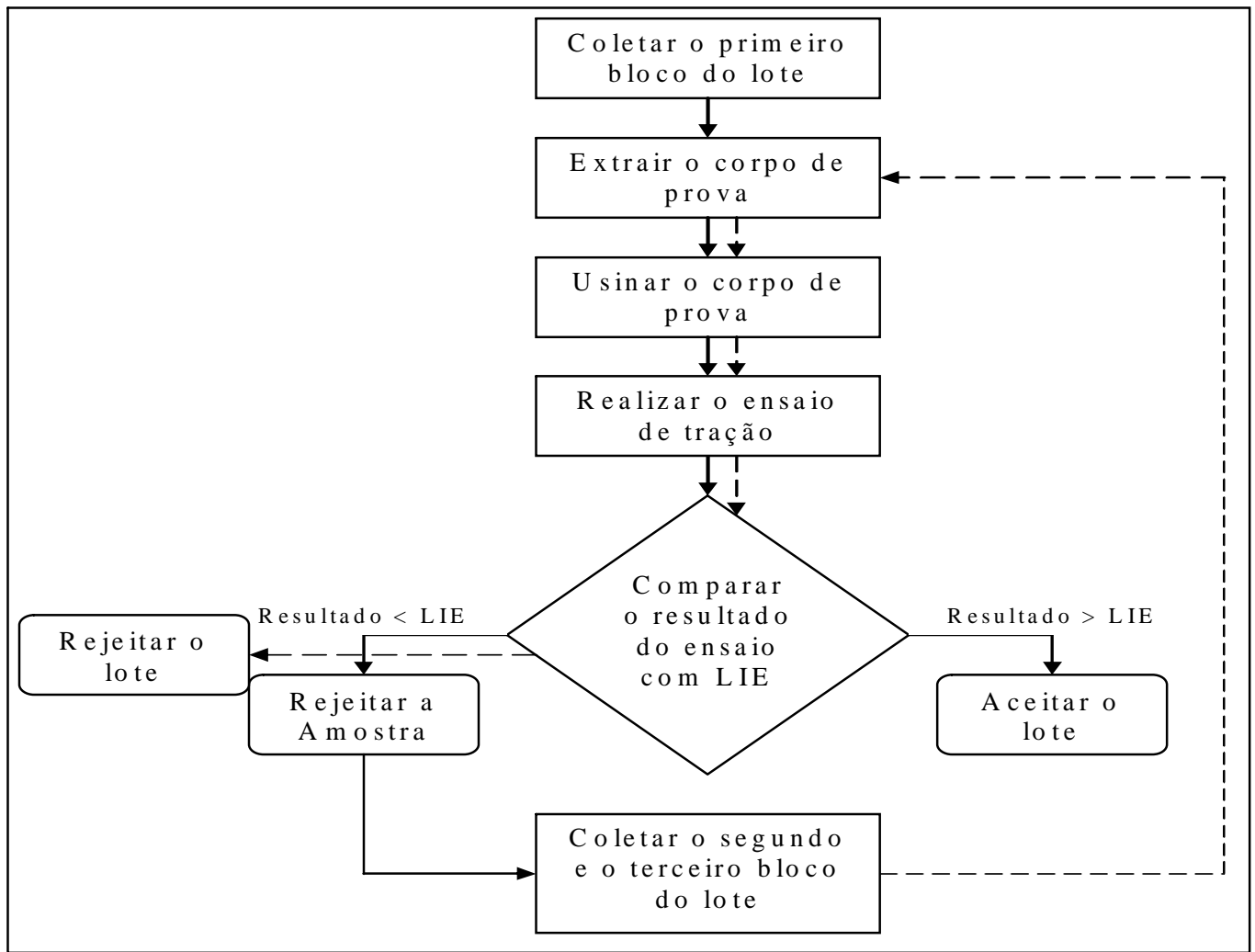

Figura 1 - Fluxograma do Processo de Inspeção.

Fonte: Autores.

\section{APRESENTAÇÃO DOS DADOS}

Os dados dos resultados dos testes de tração fornecidos pela empresa, foram acessados através do Núcleo de Normalização e Qualimetria (NNQ) da Universidade Federal de Santa Catarina (UFSC), os quais correspondem a ensaios de tração realizados na linha de fundição, sempre que ocorreram produções, durante todo o ano de 2004. Estes dados estão apresentados na sua forma original no quadro 1, possuindo freqüência amostral de um bloco/dia. 
Quadro 1: Tração de Blocos (Mpa)

\begin{tabular}{|c|c|c|c|c|c|c|c|c|c|c|c|c|c|}
\hline Amostra & Tração & Amostra & Tração & Amostra & Tração & Amostra & Tração & Amostra & Tração & Amostra & Tração & Amostra & Tração \\
\hline 1 & 279 & 29 & 251 & 57 & 252 & 85 & 231 & 113 & 245 & 141 & 262 & 169 & 261 \\
\hline 2 & 256 & 30 & 253 & 58 & 261 & 86 & 250 & 114 & 247 & 142 & 250 & 170 & 256 \\
\hline 3 & 266 & 31 & 241 & 59 & 239 & 87 & 273 & 115 & 238 & 143 & 268 & 171 & 259 \\
\hline 4 & 267 & 32 & 269 & 60 & 243 & 88 & 278 & 116 & 252 & 144 & 260 & 172 & 263 \\
\hline 5 & 248 & 33 & 255 & 61 & 270 & 89 & 282 & 117 & 259 & 145 & 246 & 173 & 265 \\
\hline 6 & 259 & 34 & 250 & 62 & 253 & 90 & 270 & 118 & 269 & 146 & 237 & 174 & 257 \\
\hline 7 & 264 & 35 & 258 & 63 & 240 & 91 & 264 & 119 & 254 & 147 & 264 & 175 & 262 \\
\hline 8 & 250 & 36 & 252 & 64 & 284 & 92 & 251 & 120 & 274 & 148 & 265 & 176 & 271 \\
\hline 9 & 247 & 37 & 249 & 65 & 258 & 93 & 248 & 121 & 274 & 149 & 267 & 177 & 283 \\
\hline 10 & 264 & 38 & 260 & 66 & 235 & 94 & 252 & 122 & 272 & 150 & 274 & 178 & 247 \\
\hline 11 & 263 & 39 & 232 & 67 & 258 & 95 & 262 & 123 & 278 & 151 & 282 & 179 & 251 \\
\hline 12 & 251 & 40 & 253 & 68 & 252 & 96 & 261 & 124 & 245 & 152 & 256 & 180 & 256 \\
\hline 13 & 238 & 41 & 251 & 69 & 278 & 97 & 269 & 125 & 267 & 153 & 269 & 181 & 237 \\
\hline 14 & 235 & 42 & 255 & 70 & 237 & 98 & 241 & 126 & 263 & 154 & 255 & 182 & 240 \\
\hline 15 & 248 & 43 & 250 & 71 & 260 & 99 & 255 & 127 & 252 & 155 & 260 & 183 & 258 \\
\hline 16 & 266 & 44 & 267 & 72 & 272 & 100 & 249 & 128 & 269 & 156 & 270 & 184 & 255 \\
\hline 17 & 276 & 45 & 257 & 73 & 264 & 101 & 239 & 129 & 270 & 157 & 255 & 185 & 253 \\
\hline 18 & 239 & 46 & 247 & 74 & 265 & 102 & 251 & 130 & 263 & 158 & 267 & - & - \\
\hline 19 & 264 & 47 & 253 & 75 & 268 & 103 & 256 & 131 & 230 & 159 & 265 & - & - \\
\hline 20 & 246 & 48 & 245 & 76 & 283 & 104 & 261 & 132 & 253 & 160 & 266 & - & - \\
\hline 21 & 267 & 49 & 265 & 77 & 253 & 105 & 281 & 133 & 251 & 161 & 258 & - & - \\
\hline 22 & 257 & 50 & 252 & 78 & 255 & 106 & 263 & 134 & 235 & 162 & 247 & - & - \\
\hline 23 & 248 & 51 & 276 & 79 & 277 & 107 & 285 & 135 & 260 & 163 & 257 & - & - \\
\hline 24 & 246 & 52 & 270 & 80 & 256 & 108 & 247 & 136 & 264 & 164 & 253 & - & - \\
\hline 25 & 268 & 53 & 261 & 81 & 273 & 109 & 271 & 137 & 262 & 165 & 272 & - & - \\
\hline 26 & 274 & 54 & 263 & 82 & 277 & 110 & 259 & 138 & 272 & 166 & 249 & - & - \\
\hline 27 & 257 & 55 & 264 & 83 & 247 & 111 & 245 & 139 & 242 & 167 & 269 & - & - \\
\hline 28 & 257 & 56 & 254 & 84 & 265 & 112 & 264 & 140 & 251 & 168 & 250 & - & - \\
\hline
\end{tabular}

Fonte: Empresa.

\section{ESTUDOS PRELIMINARES}

Para que se possa ter uma visão geral do comportamento estatístico destes dados e as possibilidades de aplicação de conceitos específicos de CEP, foram realizados testes objetivando verificar a presença de normalidade na distribuição da série e/ou a existência de autocorrelação serial. Foram também realizadas estimativas da média e do desvio padrão dos resultados coletadas ao longo dos 185 dias. 
Os resultados obtidos, visualizáveis nas figuras 2 e 3, foram de que os dados seguem uma distribuição normal, possibilitando, portanto, a aplicação de gráficos de controle do tipo Shewhart, e de outros gráficos alternativos que são comumente utilizados quando as amostras forem unitárias, tais como CUSUM e EWMA. A utilização dos gráficos de Shewhart se justifica pela simplicidade de construção e de interpretação dos resultados.

A introdução do conceito de gráfico de controle foi dado por Walter A. Shewhart em 1924, com o objetivo de controlar a variabilidade do processo na empresa Bell Laboratories. Particularmente o autor apresentou os gráficos de controle da média e do desvio padrão amostral. Esses gráficos consistem de uma linha central (média do processo), e duas linhas paralelas a esta, chamadas de limites inferior (LIC) e superior de controle (LSC). Todos os gráficos de controle construídos com essa mesma filosofia são denominados de gráficos de Shewhart. Infelizmente esses gráficos são ineficientes para detectar pequenas alterações na média do processo motivo pelo qual estamos aplicando outros tipos de gráficos de controle em nosso estudo.

Quanto à questão da autocorrelação, como pode ser visualizada na figura 4, a medida de tração coletada no dia t, não influenciou a medida de tração coletada no dia $\mathrm{t}+1$, significando, portanto, que o processo é de fato não autocorrelacionado. Tais características permitem que se trabalhe com os dados em sua forma original, tornando assim desnecessária qualquer transformação na série para que seja possível a aplicação da teoria e conceitos de gráficos de controle. 


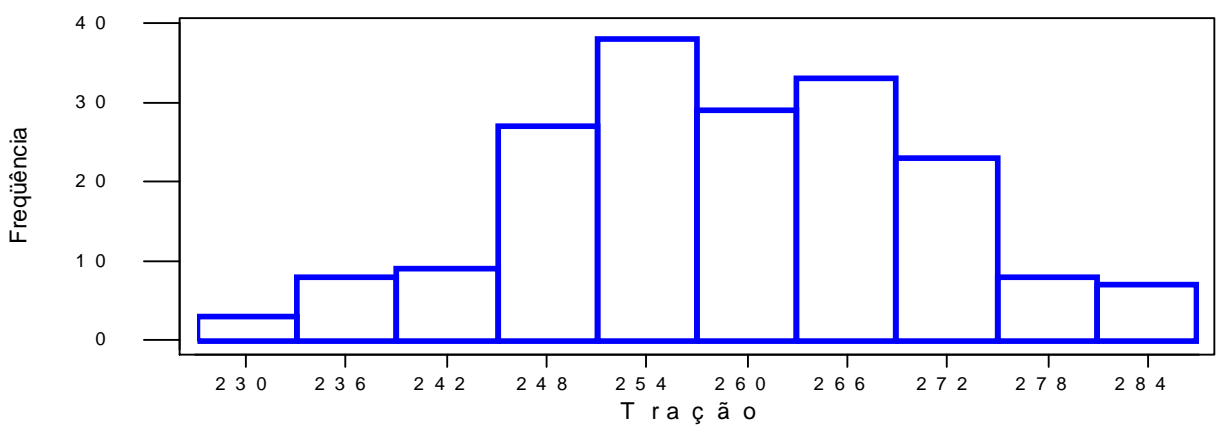

Figura 2 - Histograma.

Fonte: PcGive 10.

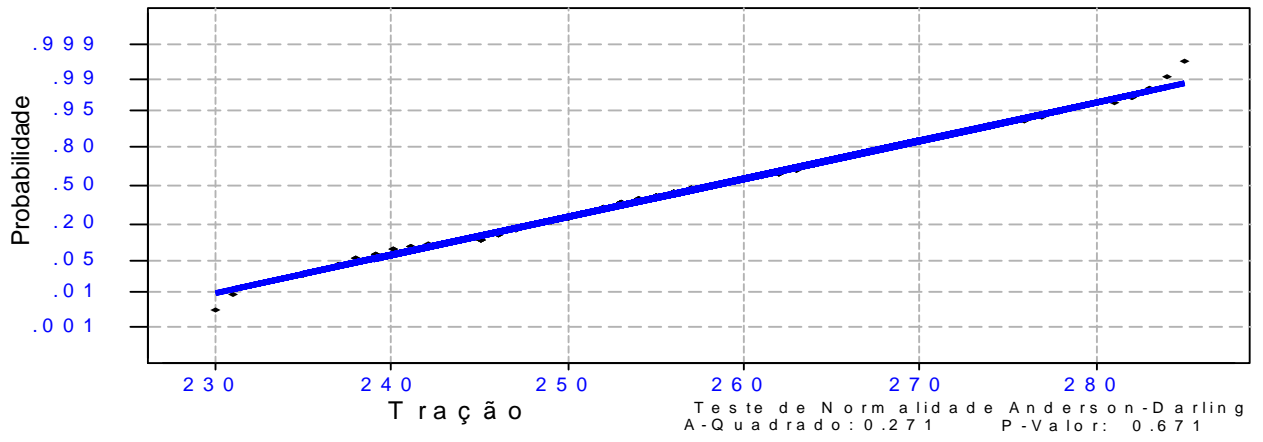

Figura 3 - Gráfico de Probabilidade da Normalidade.

Fonte: PcGive 10.

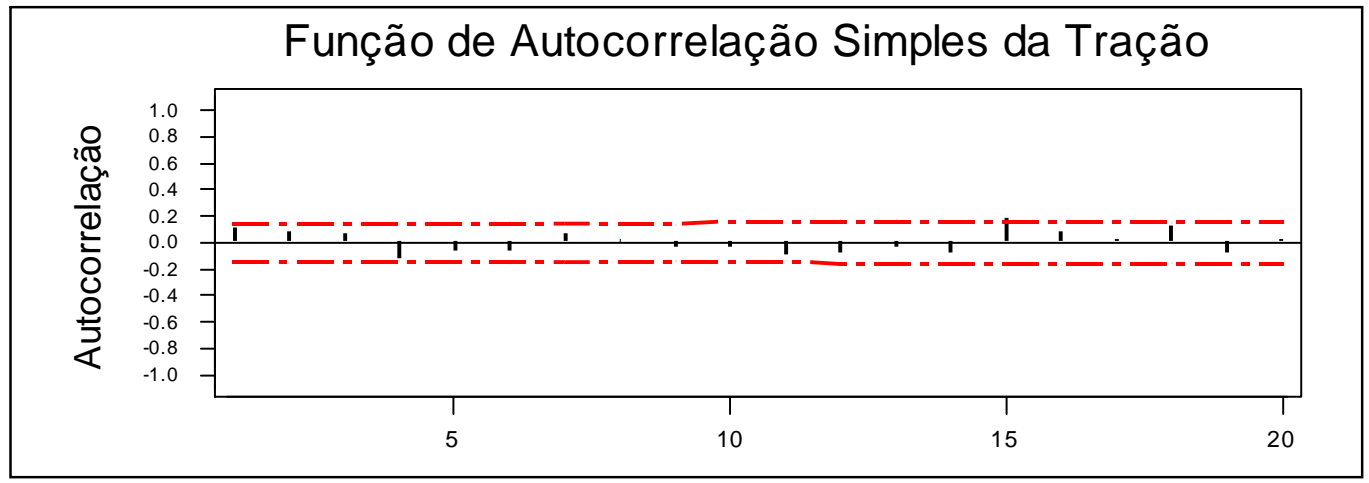

Figura 4 - Correlograma.

Fonte: PcGive 10. 
É importante e necessário mencionar que, como as amostras são de tamanho unitário, a amplitude do processo considerada neste trabalho foi à amplitude móvel, que consiste na diferença entre a maior e a menor medida de tração entre o dia t e o dia t-1. Já no caso da estimativa do desvio padrão do processo, foi realizada duas estimativas: uma através da forma tradicional (equação 3.1), considerando a amostra global, e outra através do cálculo de $S_{D}$ (equação 3.2), pois trata-se de uma medida mais confiável, por basear-se apenas na dispersão dos valores amostrais, sendo, segundo COSTA et al. (2004), insensível a causas especiais que alteram a média do processo.

Para o processo em questão, utilizando as fórmulas 3.1 e 3.2 (apresentadas a seguir), temos, respectivamente, os valores de 11.865 e 11,274. Como a estimativa a ser utilizada no restante do trabalho será a do estimador $S_{D}(11,274)$, lembramos que o valor de $\mathrm{d}_{2}$ utilizado no cálculo foi o correspondente a $\mathrm{n}=2(1,128)$, por tratar-se de uma diferença entre dois valores apenas. Já a média calculada para este processo, foi de 258,18 .

$$
\begin{aligned}
& S=\frac{1}{n-1} \sqrt{\sum_{i=1}^{n}\left(X_{i}-\bar{X}\right)^{2}} \\
& S_{D}=\frac{\overline{M R}}{d_{2}}
\end{aligned}
$$

\section{IMPLEMENTAÇÃO DE GRÁFICOS DE CONTROLE}

Objetivando detectar possíveis mudanças na média do processo, foram construídos os gráficos alternativos aos de Shewhart mais indicados para amostras unitárias, ou seja, o da soma cumulativa (CUSUM) e o da média móvel exponencialmente ponderada (EWMA - que possui desempenho similar ao da soma cumulativa). Também foi construído o gráfico da amplitude móvel, para que se conheça e analise a variabilidade do processo. As análises e interpretações feitas baseadas nestes gráficos estão apresentadas gradativamente nos tópicos a seguir. 


\subsection{Gráfico das observações individuais $\left(X_{i}\right)$}

Como não se conhecia o valor médio alvo do processo, por não ter sido informado pela empresa, antes de se construir o gráfico, foi realizado um teste de análise de variância (ANOVA). Para isto, estratificou-se a amostra em 5 sub-amostras de tamanho 37 (respeitando-se a ordem cronológica das observações coletadas), para verificar se a média do processo apresentava modificações ao longo dos 185 dias em que foram feitas as coletas amostrais.

A hipótese nula $\left(\mathrm{H}_{0}\right)$ considerada, foi de que todas as médias das sub-amostras eram estatisticamente iguais, e a hipótese alternativa $\left(\mathrm{H}_{\mathrm{a}}\right)$, de que pelo menos a média de uma das sub-amostras diferia das demais. Como pode ser visualizado na figura 5 , onde são apresentados os resultados do teste ANOVA, não se obteve fortes evidências para que se rejeitasse a hipótese nula, levando-nos a concluir com nível de significância de 5\%, que a média do processo realmente não sofreu alterações significativas ao longo do período analisado.

\begin{tabular}{|c|c|c|c|c|}
\hline \multicolumn{5}{|c|}{ Análise de Variância } \\
\hline Fonte & $\mathrm{DF}$ & SS & MS & $\mathrm{F}$ \\
\hline Fator & 5 & 606 & 121 & 0.507 \\
\hline Erro & 364 & 51198 & 141 & \\
\hline \multirow[t]{2}{*}{ Total } & 369 & 51804 & & \\
\hline & & & & $\begin{array}{l}\text { Individual 95\% CIs For Mean } \\
\text { Based on Pooled StDev }\end{array}$ \\
\hline Level & $\mathrm{N}$ & Mean & StDes & -----------+----------+---------++------ \\
\hline Tração & 185 & 258.18 & 11.86 & $(----\star----)$ \\
\hline$x 1$ & 37 & 256.16 & 10.82 & $(----------*----------)$ \\
\hline$x 2$ & 37 & 256.65 & 12.12 & $(-----------\star-----------)$ \\
\hline$\times 3$ & 37 & 260.76 & 13.59 & $(------------\star------------)$ \\
\hline $\mathrm{x} 4$ & 37 & 257.49 & 12.24 & $(-----------\star------------)$ \\
\hline$x 5$ & 37 & 259.86 & 10.21 & $\left(---------{ }^{*}----------\right)$ \\
\hline
\end{tabular}

Figura 5 - Resultados da ANOVA.

Fonte: PcGive 10.

Apesar dos resultados do teste ANOVA apresentados mostrarem que não houve uma diferença estatisticamente significativa entre as médias das sub-amostras, sob o ponto de vista do controle estatístico de processo, na terceira sub-amostra identificada como X3 (entre a $75^{\mathrm{a}}$ e a $111^{\mathrm{a}}$ observação) ocorreu uma sensível 
modificação na média do processo, o que pode ser mais bem visualizado través da figura 6.

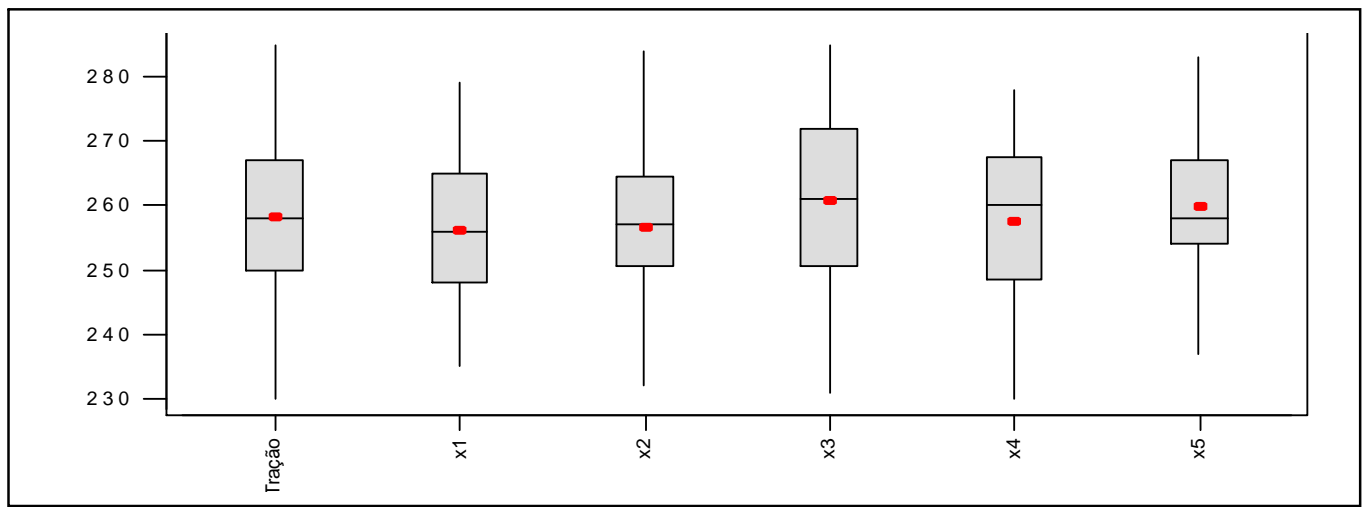

Figura 6 - Box-plots da Tração Versus as Sub-amostras.

Fonte: PcGive 10 .

Na figura 7 é apresentado o gráfico dos valores individuais das 37 primeiras observações (sub-amostra X1), no qual foram inseridas posteriormente as demais observações, para que fosse possível identificar se alguma das observações pertencentes a qualquer uma das sub-amostras se apresentaria fora dos limites de controle calculados. Tal procedimento é visualizável na figura 8 , e foi adotado em todas as aplicações de gráficos de controle deste trabalho, com intuito de acompanhar tal comportamento quando se modificava o objeto de estudo em questão (média ou amplitude do processo).

Com base nas figuras 7 e 8 , pode-se concluir, num primeiro momento, que as observações individuais distribuíram-se aleatoriamente em torno da linha média do processo, mantendo-se dentro da região de controle do gráfico, não exigindo, portanto, nenhuma intervenção no processo por parte do operador. 


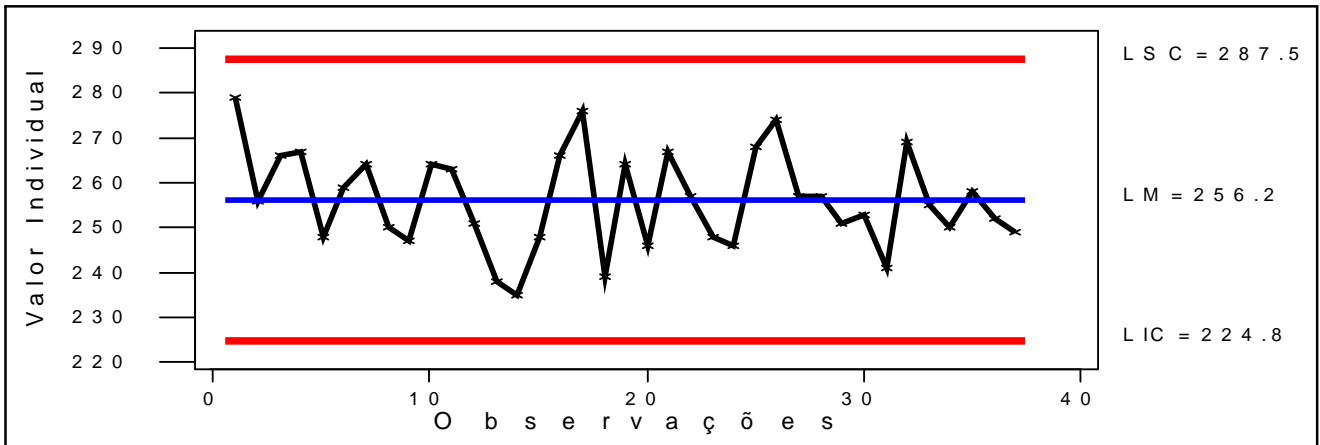

Figura 7 - Gráfico $X_{i}$ para a Sub-amostra $X_{1}$.

Fonte: PcGive 10.

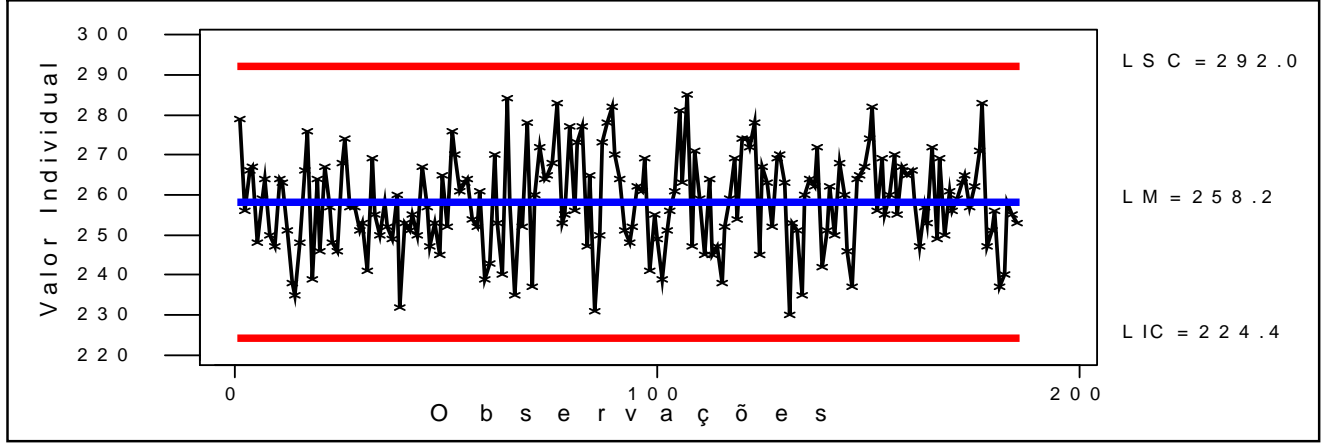

Figura 8 - Gráfico $X_{i}$ para toda a Amostra.

Fonte: PcGive 10.

\subsection{Gráfico da Amplitude Móvel (MR)}

Observando-se as figuras 9 e 10, onde a média das amplitudes recalculada automaticamente pelo software aplicativo utilizado ficou um pouco acima do valor calculado pela fórmula 3.2, pode-se concluir que de um modo geral o processo não apresentou uma variabilidade que ultrapassasse os limites de controle. No entanto, é importante mencionar que a coleta feita no sexagésimo quarto dia (sub-amostra X2) apresentou uma amplitude de $44 \mathrm{Mpa}$, portanto, fora do limite superior de controle. A ocorrência deste fato pode estar associada a duas possíveis situações mutuamente excludentes: uma relacionada à probabilidade do risco $\alpha$ (alarme falso), ou risco do produtor em rejeitar um corpo de prova usinado que seja bom, que corresponde ao 
nível de significância do processo; ou ainda a ocorrência de um alarme verdadeiro, que tenha originado uma possível intervenção no processo que eliminou a causa especial que o gerou, pois todas as amostras seguintes voltaram a ficar dentro dos limites de controle oscilando aleatoriamente em torno da linha média do processo.

É importante ressaltar que um gráfico de controle nada mais é do que uma sequiência de testes de hipóteses, em que a probabilidade do risco $\alpha$ é o erro do tipo I. Esse erro só ocorre quando se rejeita a hipótese nula verdadeira, isto é, um corpo de prova é bom, mas é rejeitado pela empresa. Mas existe também o erro do tipo II ( $\beta$ ), também conhecido como o risco do consumidor, que consiste em não rejeitar uma hipótese nula falsa. É o caso em que o corpo de prova não está dentro dos padrões especificados pela empresa, mas mesmo assim, o lote contendo todos os blocos de motores não é rejeitado como sendo problemático.

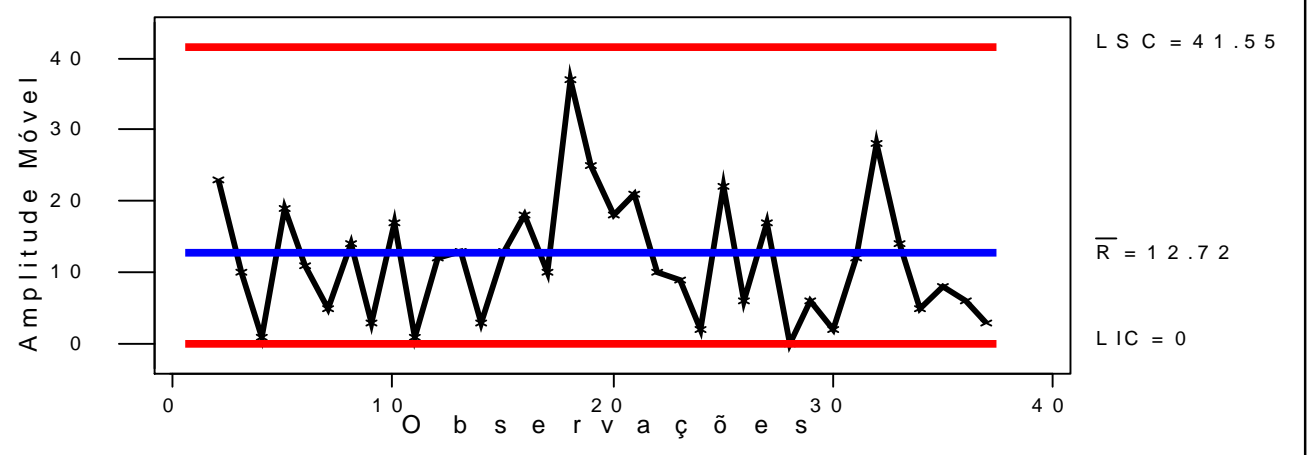

Figura 9 - Gráfico da Amplitude Móvel para a Sub-amostra $X_{1}$.

Fonte: PcGive 10. 


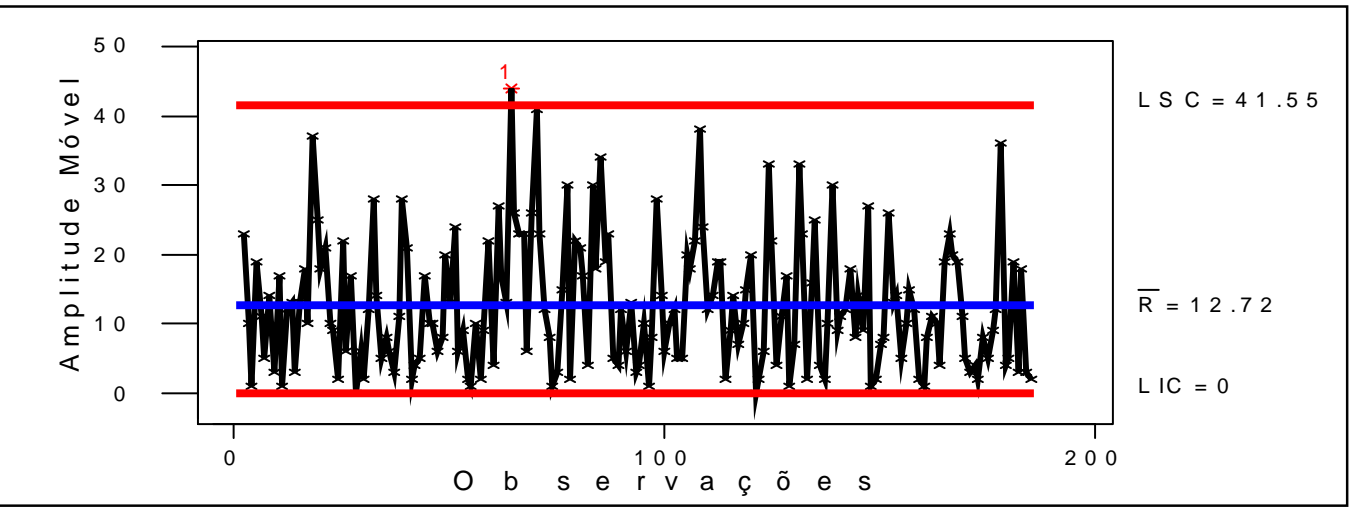

Figura 10 - Gráfico da Amplitude Móvel para toda a Amostra.

Fonte: PcGive 10.

\subsection{Gráfico da Soma Cumulativa (CUSUM)}

Os gráficos de controle de somas cumulativas foram propostos inicialmente por PAGE (1954) e posteriormente estudados por muitos autores tais como EWAN (1963), PAGE (1961), WOODALL e ADAMS (1993) dentre outros. O gráfico CUSUM incorpora diretamente toda a informação nas seqüências de valores da amostra de um valor alvo, como por exemplo, a média do processo. Além disso, o CUSUM combina informação de várias amostras, tornando-o mais eficaz do que os gráficos de Shewhart para detectar pequenas mudanças na média do processo. Outra vantagem deste tipo de gráfico sobre os de Shewhart é a sua eficiência em detectar pequenas mudanças no processo quando se utilizam amostras unitárias.

Se o objetivo de uma indústria é monitorar a média de uma determinada característica de um produto, então a forma geral do CUSUM é dada pela expressão:

$$
C_{i}=\sum_{j=1}^{i}\left(\bar{X}_{j}-\mu_{0}\right)
$$

em que $\bar{X}_{j}$ é a média da j-ésima amostra e $C_{i}$ é a soma cumulativa até, e incluindo, a i-ésima amostra. No caso de amostras de tamanho unitário, tem-se a igualdade: $\bar{X}_{j}=X_{j}$. Na prática não é muito comum utilizar a estatística definida em (4.1) para 
construir o gráfico da média, uma vez que existe um algorítmico muito eficiente denominado de CUSUM tabular, que é o mais usado. O CUSUM tabular trabalha acumulando desvios de $\mu_{0}$ que estão acima do alvo, com uma estatística $S^{+}$, e acumulando desvios de $\mu_{0}$ que estão abaixo do alvo, com uma estatística $S^{-}$. Essas estatísticas $S^{+}$e $S^{-}$são denominadas CUSUMS unilaterais superior e inferior respectivamente, e são calculadas da seguinte forma:

$$
\begin{aligned}
& S_{i}^{+}=\operatorname{máx}\left[0, X_{i}-\left(\mu_{0}+K\right)+S_{i-1}^{+}\right] \\
& S_{i}^{-}=\operatorname{máx}\left[0,\left(\mu_{0}-K\right)-X_{i}+S_{i-1}^{-}\right],
\end{aligned}
$$

onde os valores inicias são $S_{i}^{+}=S_{i}^{-}=0$. A constante $K$ é conhecida como valor de referência ou de tolerância, e é sempre escolhido a meio caminho entre o valor-alvo $\mu_{0}$ e o valor da média com o processo fora de controle $\mu_{1}$. Se mudança na média é expressa em unidade de desvios-padrão, como por exemplo, $\mu_{1}=\mu_{0}+\delta \sigma$, então o valor de $K$ é a metade da magnitude da mudança, isto é:

$$
K=\frac{\delta}{2} \sigma=\frac{\left|\mu_{1}-\mu_{0}\right|}{2} .
$$

A região de atuação do gráfico ou intervalo de decisão $H$, é outra constante que precisa ser estimada e o mais comum é adotar $H$ igual a 5 vezes o desvio padrão $\sigma$ do processo.

Como desejávamos detectar pequenas mudanças na média do processo, optamos por empregar o gráfico CUSUM em nosso estudo de caso. Observe-se nas figuras 11 e 12 a ocorrência de uma pequena mudança na média do processo (já detectada na figura 6) que muito provavelmente está ligada à ocorrência de uma causa verdadeira que foi rapidamente identificada e combatida, fazendo com o que o processo voltasse, já na próxima coleta, a região de controle do gráfico. Como o sinal disparado pelo gráfico CUSUM ocorreu na $91^{\text {a }}$ observação, podemos concluir que o 
momento do descontrole (MD) ocorreu aproximadamente na $86^{\mathrm{a}}$ observação, dado que o alarme disparou após cinco desvios positivos consecutivos da média do processo.

Tais gráficos, da forma como foram construídos, nos permitem também, contemplar tanto a estatística $S_{i}^{+}$quanto à $S_{i}^{-}$. Com relação a $K$ e $H$, utilizamos, respectivamente, os valores 5 e 0,5 , buscando manter o intervalo médio entre alarmes falsos igual a 370 amostras, conforme os gráficos originais de Shewhart. Esse intervalo de 370 amostras é também chamado de ARL (Average Run Length), ou seja, trata-se do número médio de amostras observadas até a ocorrência um alarme (NMA).

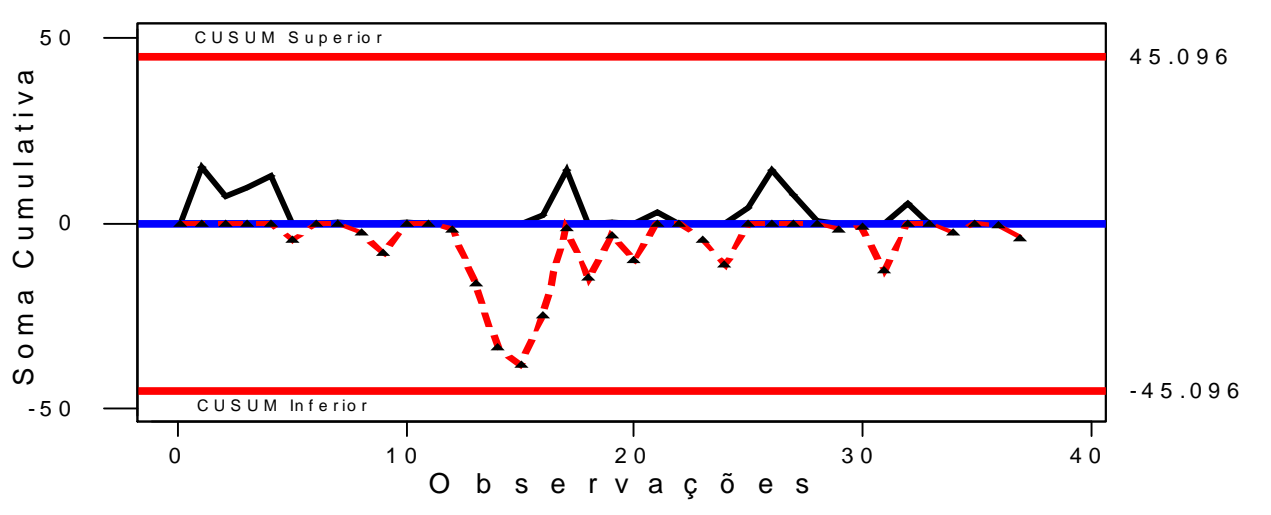

Figura 11 - Gráfico CUSUM para a Sub-amostra $\mathrm{X}_{1}$.

Fonte: PcGive 10.

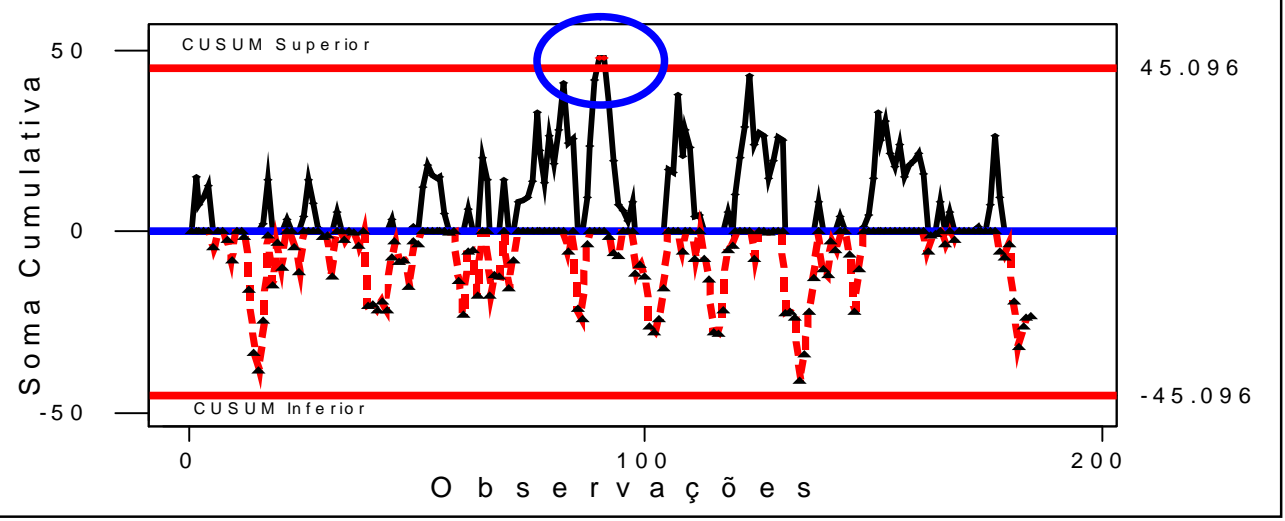

Figura 12 - Gráfico CUSUM para toda a Amostra.

Fonte: PcGive 10. 


\subsection{Gráfico da Média Móvel Exponencialmente Ponderada (EWMA)}

Similarmente ao gráfico da soma cumulativa, o gráfico da média móvel exponencialmente ponderada, é uma forma alternativa ao gráfico $\bar{X}$ de Shewhart, utilizado para detectar pequenas mudanças na média do processo. A principal diferença entre estes dois gráficos, é que no EWMA os dados não se afastam gradual e indefinidamente da linha média como no caso do CUSUM, e sim, apenas até atingir a nova média, passando então a oscilar aleatoriamente em torno da mesma.

O gráfico da média móvel exponencialmente ponderada foi introduzido por ROBERTS (1959) e foi estudado por muitos pesquisadores dentre os quais se destacaram CROWDER (1989) e LUCAS e SACCUCCI (1990). Roberts definiu o gráfico utilizando a seguinte expressão:

$$
Z_{i}=\lambda X_{i}+(1-\lambda) Z_{i-1},
$$

em que $0<\lambda \leq 1$ é uma constante e o valor inicial do EWMA (exigido com a primeira amostra em $i=1$ ) é o alvo do processo, de modo que $Z_{0}=\mu_{0}$. O desempenho desse gráfico depende tanto dos valores múltiplos do desvio padrão, quanto dos valores de $\lambda$. De modo geral, o gráfico EWMA apresenta desempenho similar ao gráfico CUSUM, com os valores de $\lambda$ variando no intervalo $0,05 \leq \lambda \leq 0,25$, sendo que na prática os valores mais utilizados são: $\lambda=0,05$, $\lambda=0,10$ e $\lambda=0,20$. Duas vantagens desse gráfico são: ( $i)$ pode ser utilizado para fazer previsões de valores futuros e (ii) é robusto à não normalidade dos dados. $\mathrm{O}$ gráfico EWMA é construído, fazendo-se a plotagem de $Z_{i}$ versus o número da amostra $i$ (ou tempo).

A utilização desse gráfico em nosso estudo de caso, foi de grande valia para mostrar as pequenas mudanças e as oscilações sofridas pela média do processo. Observando as figuras 13 e 14 notam-se sutis modificações na média do processo, como já detectado no gráfico das somas cumulativas, pois existem oscilações da média ao longo do processo acima e abaixo de 258.18 , que representa a média geral estimada. 


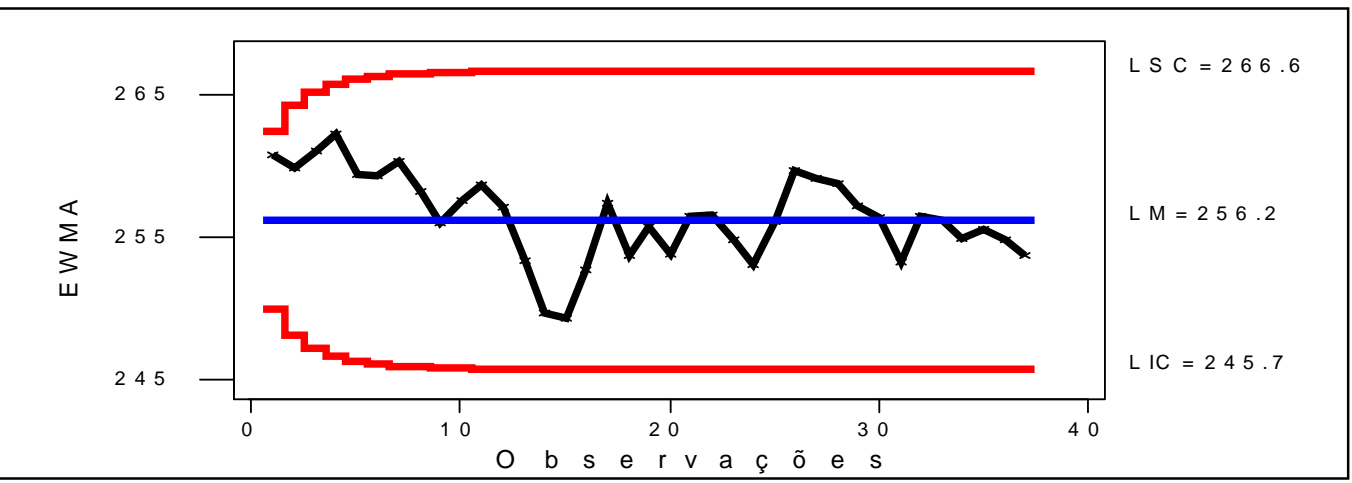

Figura 13 - Gráfico EWMA para a Sub-amostra $X_{1}$.

Fonte: PcGive 10.

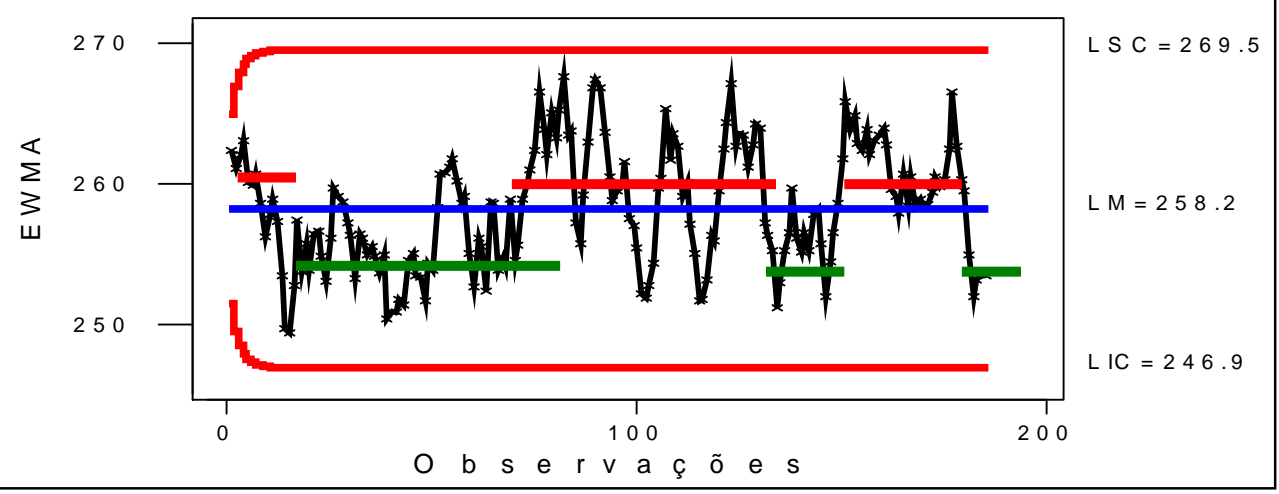

Figura 14 - EWMA para toda a Amostra.

Fonte: PcGive 10.

\section{ANÁLISE DOS ÍNDICES DE CAPACIDADE DO PROCESSO}

Toda a análise feita até o momento buscou identificar informações a respeito da estabilidade do processo, ou seja, se o mesmo estava funcionando adequadamente. Porém, torna-se necessário sabermos também se o mesmo está produzindo uma porcentagem significativa de blocos fora dos limites de especificação, isto é, se está produzindo itens defeituosos, pois segundo COSTA et al. (2004), não existe uma relação direta entre estabilidade e capacidade, podendo um processo mesmo estando em controle, produzir itens fora dos limites de especificação e vice-versa. 
Para os resultados dos testes de tração fornecidos pela empresa, como já mencionado, só possuem limite inferior de especificação, portanto, foi utilizado como parâmetro adimensional para medir sua capacidade o índice Cpk (equação 4.6), pois dos mais utilizados é o único que permite calcular a capacidade unilateralmente. $\mathrm{Na}$ figura 15, é possível observar que o processo é incapaz, segundo o cálculo do Cpk, pois seu valor calculado ficou abaixo de 1. Isto nos leva a concluir que o processo realizado no ano de 2004 na empresa, esteve na maior parte do tempo em controle, porém, mostrou-se incapaz, produzindo uma porcentagem significativa de itens defeituosos.

$$
C p_{k}=\frac{\mu-L I E}{3 \sigma}
$$

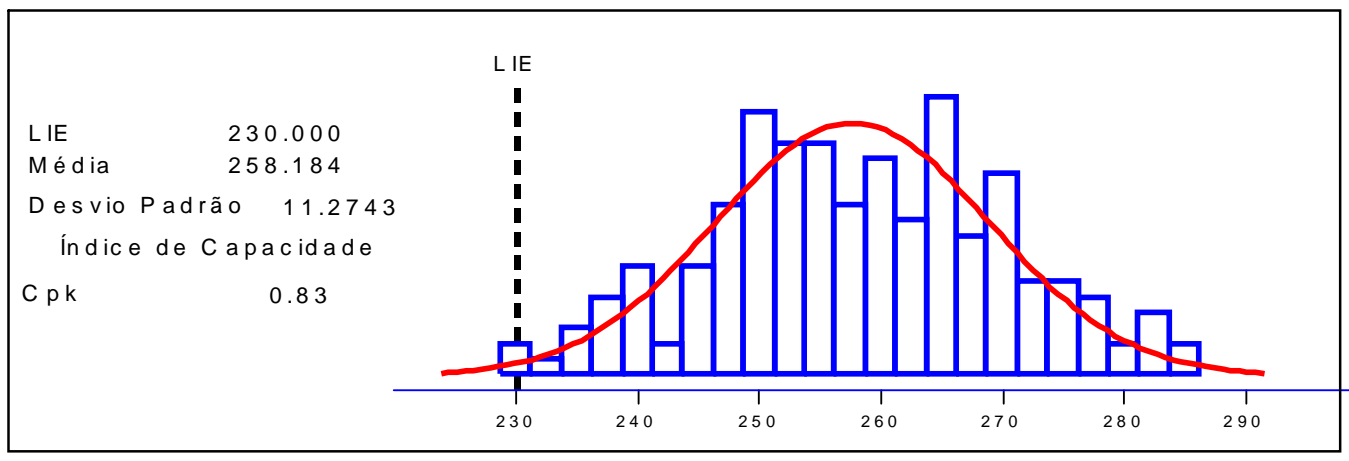

Figura 15 - Análise da Capacidade do Processo - Dados Originais.

Fonte: PcGive 10.

\section{Conclusões}

Com relação à qualidade da produção de blocos para motores de caminhão, podemos concluir que a empresa trabalha com um processo estável, porém, ficou evidenciada a presença de pequenas oscilações em torno da média, como mostrado nos gráficos EWMA e CUSUM. Identificou-se também que o processo estudado apresenta uma variabilidade que não pode ser desconsiderada como mostrado nas 
figuras 9 e 10, onde existem várias observações muito próximas dos limites de controle o que é reforçado pela figura 14. Nessa figura, se observa diversas variações da média ao longo dos 185 dias em que foi monitorado, o que segundo MONTGOMERY (2004), significa uma falta de qualidade, na medida em que qualidade é inversamente proporcional à variabilidade.

A causa desta variabilidade, muito possivelmente está ligada a um procedimento característico no processo de fundição de ferro, que consiste na recomposição manual dos componentes químicos responsáveis por dar a liga ideal ao material, perdidos durante o processo de re-fundição, como ocorre, por exemplo, com blocos rejeitados ou utilizados no processo de inspeção.

Por outro lado, confiando-se, antes de tudo, nas informações retiradas dos próprios dados, o cálculo do índice de capacidade indicou uma aparente incongruência, uma vez que, como pode ser observado nos dados do quadro 1, todas as observações são maiores que o limite inferior de especificação (LIE) informado pela empresa (230 Mpa), com exceção da observação de número 131, cujo valor é exatamente igual ao LIE. Esta situação leva a se esperar verificar que o processo fosse pelo menos razoavelmente capaz ao invés de incapaz, como apresentado na figura 15.

Mas a resposta para essa aparente incongruência está intrinsecamente relacionada à já mencionada não pequena variabilidade do processo, pois o cálculo do índice de capacidade Cpk dado pela equação (4.6), faz com que seu valor estimado seja "infectado" negativamente por esta variabilidade.

Os resultados obtidos com a utilização dos diversos gráficos de controle para amostras de tamanho unitário, juntamente com a estimação do índice de capacidade, atendeu de forma razoavelmente satisfatória às expectativas dos autores. A frustração ficou por conta de se esperar encontrar um processo estatisticamente capaz de produzir os blocos dentro das especificações desejadas, uma vez que se trata de uma grande empresa e já que trabalha há muito tempo com esse tipo de produto.

Como considerações finais, recomendamos a possibilidade de implementação de um controle estatístico no processo de recomposição dos componentes químicos responsáveis pela liga do ferro fundido. Acredita-se que esta iniciativa poderia levar a 
uma redução significativa da variabilidade do processo do bloco analisado, tendo em vista que é um dos possíveis fatores que influencia nos resultados dos testes de tração do mesmo.

\section{Agradecimentos}

Ao Núcleo de Normalização e Qualimetria (NNQ) do Departamento de Engenharia de Produção e Sistemas (DEPS) que colocou a disposição toda sua estrutura física, incluindo o acesso ao software PcGive 10. Também ao Conselho Nacional de Desenvolvimento Científico e Tecnológico CNPq - Brasil, pelo apoio concedido através de bolsa de doutorado.

\section{REFERÊNCIAS}

COSTA, A. F. B., EPPRECHT, E.K., CARPINETTI, L.C.R. Controle Estatístico de Qualidade. 1 ed. São Paulo: Editora Atlas, 2004.

CROWDER, S. V. "Design of Exponentially Weighted Moving Average Schemes,” Journal of Quality Technology, Vol. 19, 1989.

EWAN, W. D. "When and How to Use Cu-Sum Charts," Technometrics, Vol. 5, 1963.

LUCAS, J. M.; SACCUCCI, M. S. "Exponentially Weighted Moving Average Control Schemes: Properties and Enhancements ," Techometrics, Vol. 32, 1990. 
MONTGOMERY, D.C. Introduction to Statistical Quality Control. 4 ed. Rio de Janeiro: LTC - Livros Técnicos e Científicos Editora S.A., 2004.

PAGE, E. S. “Continuous Inspection Schemes,” Biometrics, Vol. 41, 1954.

PAGE, E. S. “Cumulative Sum Control Charts,” Technometrics, Vol. 3, 1961.

ROBERTS, S. W. "Properties of Chart Tests Based on Geometric Moving Average," Technometrics, Vol. 1, 1959.

SAMOHYL, R.W. Médias por Amortecimento Exponencial (MAE). Disponível em: www.webensino.ufsc.br Acesso em: 31/03/2004.

SAMOHYL, R.W. Introdução a Covariância e Correlação. Disponível em: www.webensino.ufsc.br Acesso em: 25/04/2004.

WOODAL, W. H.; ADAMS, B. M. "The Statistical Design of CUSUM Charts," Quality Engineering, Vol. 5, '993.

Artigo recebido em 31/05/2006 e publicado em 21/08/2007. 